\title{
A sufficient condition for a Hibi ring to be level and levelness of Schubert cycles
}

\author{
Mitsuhiro MIYAZAKI \\ Dept. Math., Kyoto University of Education, \\ Fukakusa-Fujinomori-cho, Fushimi-ku, Kyoto, 612-8522 Japan \\ E-mail: g53448@kyokyo-u.ac.jp
}

\begin{abstract}
Let $K$ be a field, $D$ a finite distributive lattice and $P$ the set of all join-irreducible elements of $D$. We show that if $\{y \in P \mid y \geq x\}$ is pure for any $x \in P$, then the Hibi ring $\mathcal{R}_{K}(D)$ is level. Using this result and the argument of sagbi basis theory, we show that the homogeneous coordinate rings of Schubert subvarieties of Grassmannians are level. MSC:13F50, 13H10, 13A02, 14M15, 13P10
\end{abstract}

\section{Introduction}

Let $K$ be a field and $D$ a finite distributive lattice. Hibi Hib defined the ring $\mathcal{R}_{K}(D)$, which is now called the Hibi ring. He showed that $\mathcal{R}_{K}(D)$ is an algebra with straightening law (ASL for short) over $K$ generated by $D$ and is a normal affine semigroup ring. So by the result of Stanley [Sta2], the canonical module of $\mathcal{R}_{K}(D)$ is described combinatorially.

On the other hand, Grassmannians and their Schubert subvarieties are important and fascinating objects of algebraic geometry and commutative algebra. And their homogeneous coordinate rings are extensively studied. For example, it is known that they are normal Cohen-Macaulay domains and the homogeneous coordinate rings of Grassmannians are Gorenstein. The characterization of the Gorenstein property of the homogeneous coordinate ring of a Schubert variety (Schubert cycle for short) is also known. Note also, that the combinatorial study of these rings are the origin of the theory of ASL.

On the other hand, Stanley Sta1] defined the notion called level for standard graded algebras. This is a notion between Cohen-Macaulay property and Gorenstein property for standard graded algebras.

In this article, we give a sufficient condition for $\mathcal{R}_{K}(D)$ to be level. We also show that the Schubert cycles can be realized as a subring of a polynomial ring which have finite homogeneous sagbi basis. And the initial algebra of these rings are Hibi rings satisfying the sufficient condition given in this article. So the standard deformation argument shows that every Schubert cycle is level. 


\section{Preliminaries}

In this article, all rings and algebras are commutative with identity element.

We first recall the results and notation of Hibi [Hib], with a few modication.

We denote by $\boldsymbol{N}$ the set of all non-negative integers.

Let $P$ be a finite partially ordered set (poset for short).

The length of a chain (totally ordered subset) $X$ of $P$ is $\# X-1$, where $\# X$ is the cardinality of $X$.

The rank of $P$, denoted by $\operatorname{rank} P$, is the maximum of the lengths of chains in $P$.

A poset is said to be pure if its all maximal chains have the same length.

The height (resp. coheight) of an element $x \in P$, denoted by $\operatorname{ht}_{P} x$ or simply ht $x\left(\operatorname{resp} . \operatorname{coht}_{P} x\right.$ or $\left.\operatorname{coht} x\right)$, is the rank of $\{y \in P \mid y \leq x\}$ (resp. $\{y \in P \mid y \geq x\}$ ).

A poset ideal of $P$ is a subset $I$ of $P$ such that $x \in I, y \in P$ and $y \leq x$ imply $y \in I$.

For $x, y \in P, y$ covers $x$, denoted by $x<y$, means $x<y$ and there is no $z \in P$ such that $x<z<y$.

We denote by $\widehat{P}$ the extended poset $P \cup\{\infty,-\infty\}$ where $\infty$ and $-\infty$ are new elements and $-\infty<x<\infty$ for any $x \in P$.

A map $\nu: \widehat{P} \rightarrow \boldsymbol{N}$ is order reversing if $x \leq y$ in $\widehat{P}$ implies $\nu(x) \geq \nu(y)$ and strictly order reversing if $x<y$ in $\widehat{P}$ implies $\nu(x)>\nu(y)$.

The set of all order reversing maps (resp. strictly order reversing maps) from $\widehat{P}$ to $N$ which map $\infty$ to 0 is denoted by $\overline{\mathcal{T}}(P)(\operatorname{resp} . \mathcal{T}(P))$.

Now let $D$ be a finite distributive lattice and $K$ a field. A join-irreducible element in $D$ is an element in $D$ which covers exactly one element in $D$. Recall the result of Birkhoff $[\mathrm{Bir}$. Let $P$ be the set of all join-irreducible elements in $D$. Then $D$ is isomorphic to $J(P)$ ordered by inclusion, where $J(P)$ is the set of all poset ideals of $P$. The isomorphisms $\Phi: D \rightarrow J(P)$ and $\Psi: J(P) \rightarrow D$ are given by

$$
\begin{array}{ll}
\Phi(\alpha):=\{x \in P \mid x \leq \alpha \text { in } D\} & \text { for } \alpha \in D \text { and } \\
\Psi(I):=\bigvee_{x \in I} x & \text { for } I \in J(P)
\end{array}
$$

where empty join is defined to be the minimal element of $D$.

Let $\left\{T_{x}\right\}_{x \in \widehat{P}}$ and $\left\{X_{\alpha}\right\}_{\alpha \in D}$ be families of indeterminates. Hibi Hib] defined the ring $\mathcal{R}_{K}(D)$, which is now called the Hibi ring, by

$$
\mathcal{R}_{K}(D):=K\left[\prod_{x \in I \cup\{-\infty\}} T_{x} \mid I \in J(P)\right]
$$


as a subring of the polynomial ring $K\left[T_{x} \mid x \in \widehat{P}\right]$. He also showed that $\mathcal{R}_{K}(D)$ is isomorphic to

$$
K\left[X_{\alpha} \mid \alpha \in D\right] /\left(X_{\alpha} X_{\beta}-X_{\alpha \wedge \beta} X_{\alpha \vee \beta} \mid \alpha, \beta \in D\right) .
$$

The isomorphism is induced by the $K$-algebra homomorphism $K\left[X_{\alpha} \mid \alpha \in\right.$ $D] \rightarrow K\left[T_{x} \mid x \in \widehat{P}\right]$ sending $X_{\alpha}$ to $T_{-\infty} \prod_{x \leq \alpha} T_{x}$. And $\mathcal{R}_{K}(D)$ is a graded ASL over $K$ generated by $D$.

It is easily verified that $\mathcal{R}_{K}(D)$ is an affine semigroup ring such that

$$
\mathcal{R}_{K}(D)=\bigoplus_{\nu \in \overline{\mathcal{T}}(P)} K\left(\prod_{x \in \widehat{P}} T_{x}^{\nu(x)}\right) .
$$

So by the result of Hocster $\left[\mathrm{Hoc}, \mathcal{R}_{K}(D)\right.$ is a normal Cohen-Macaulay domain. And by the result of Stanley Sta2],

$$
\bigoplus_{\nu \in \mathcal{T}(P)} K\left(\prod_{x \in \widehat{P}} T_{x}^{\nu(x)}\right)
$$

is the canonical module of $\mathcal{R}_{K}(D)$.

\section{A sufficient condtion for $\mathcal{R}_{K}(D)$ to be level}

Let $D$ be a finite distributive lattice and let $P$ be the set of all join-irreducible elements in $D$. We introduce the homogeneous grading on $\mathcal{R}_{K}(D) \simeq K\left[X_{\alpha} \mid\right.$ $\alpha \in D] /\left(X_{\alpha} X_{\beta}-X_{\alpha \wedge \beta} X_{\alpha \vee \beta} \mid \alpha, \beta \in D\right)$ by setting $\operatorname{deg} X_{\alpha}=1$ for any $\alpha \in D$. Then $\mathcal{R}_{K}(D)$ is a standard graded algebra, that is, a Noetherian graded algebra over a field generated by elements of degree 1 .

Stanley [Sta1] defined the level property for standard graded algebras.

Definition 3.1 Let $A=\bigoplus_{n>0} A_{n}$ be a Cohen-Macaulay standard graded $K$-algebra, and let $\left(h_{0}, \ldots, h_{s}\right), h_{s} \neq 0$ be the $h$-vector of $A$. Then we say that $A$ is level if $h_{s}=\operatorname{type} A$.

Suppose that $\operatorname{dim}_{K} A_{1}=n$ and $\operatorname{dim} A=d$ in the notation above, and $S \rightarrow A$ is a natural $K$-algebra epimorphism, where $S$ is a polynomial ring over $K$ with $n$ variables. Then the following fact is easily verified.

Lemma 3.2 The following conditions are equivalent.

(1) A is level.

(2) The degree of the generators of the canonical module of $A$ is constant. 
(3) If $F_{\bullet}$ is the minimal $S$-free resolution of $A$, then the degree of the generators of $F_{n-d}$ is constant.

A Hibi ring is not necessarily level as the example of Hibi [Hib, $\S 1$ e) Example] shows. But we have got the following sufficient condition for $\mathcal{R}_{K}(D)$ to be level.

Theorem 3.3 Assume that $\{y \in P \mid y \geq x\}$ is pure for any $x \in P$, then $\mathcal{R}_{K}(D)$ is level.

Note that the condition on $P$ above is equivalent to the following: for any $x$, $y \in P$ with $x<\cdot y, \operatorname{coht}_{\widehat{P}} x=\operatorname{coht}_{\widehat{P}} y+1$.

Theorem 3.3 is a direct consequence of the following lemma and the Stanley's description of the canonical module of a normal affine semigroup ring.

Lemma 3.4 In the situation of Theorem 3.3, for any $\nu \in \mathcal{T}(P)$ there is $\nu_{0} \in \mathcal{T}(P)$ such that $\nu_{0}(-\infty)=\operatorname{rank} \widehat{P}$ and $\nu-\nu_{0} \in \overline{\mathcal{T}}(P)$, where we set $\left(\nu-\nu_{0}\right)(x):=\nu(x)-\nu_{0}(x)$ for any $x \in \widehat{P}$.

proof We set $r=\operatorname{rank} \widehat{P}$ and

$$
\nu_{0}(x):=\max \left\{\operatorname{coht}_{\widehat{P}} x, r-\nu(-\infty)+\nu(x)\right\}
$$

for any $x \in \widehat{P}$. Then we

Claim For any elements $x, y \in \widehat{P}$ with $x<y, \nu_{0}(x)>\nu_{0}(y)$ and $\nu(x)-$ $\nu(y) \geq \nu_{0}(x)-\nu_{0}(y)$.

We postpone the proof of the claim and finish the proof of the lemma first.

Since $\nu_{0}(x)>\nu_{0}(y)$ and $\nu(x)-\nu(y) \geq \nu_{0}(x)-\nu_{0}(y)$ for any $x, y \in \widehat{P}$ with $x<y$, it is easily verified that, $\nu_{0}(x)>\nu_{0}(y)$ and $\nu(x)-\nu(y) \geq \nu_{0}(x)-\nu_{0}(y)$ for any $x, y \in \widehat{P}$ with $x<y$. And $\nu_{0}(\infty)=0$ by definition. So $\nu_{0} \in \mathcal{T}(P)$. And $\nu(x)-\nu_{0}(x) \geq \nu(y)-\nu_{0}(y) \geq \nu(\infty)-\nu_{0}(\infty)=0$ for any $x, y \in \widehat{P}$ with $x<y$. Therefore $\nu-\nu_{0} \in \overline{\mathcal{T}}(P)$. Note $\nu_{0}(-\infty)=r$ by definition.

Now we prove the claim. Consider the case where $\operatorname{coht}_{\widehat{P}} x \leq r-\nu(-\infty)+$ $\nu(x)$ first. Since $\operatorname{coht}_{\widehat{P}} y \leq \operatorname{coht}_{\widehat{P}} x-1$ and $\nu(y)<\nu(x)$, we see that

$$
\operatorname{coht}_{\widehat{P}} y<\operatorname{coht}_{\widehat{P}} x \leq r-\nu(-\infty)+\nu(x)
$$

and

$$
r-\nu(-\infty)+\nu(y)<r-\nu(-\infty)+\nu(x) .
$$


Therefore

$$
\begin{aligned}
\nu_{0}(x) & =r-\nu(-\infty)+\nu(x) \\
& >\max \left\{\operatorname{coht}_{\widehat{P}} y, r-\nu(-\infty)+\nu(y)\right\} \\
& =\nu_{0}(y)
\end{aligned}
$$

and

$$
\begin{aligned}
\nu(x)-\nu(y) & =(r-\nu(-\infty)+\nu(x))-(r-\nu(-\infty)+\nu(y)) \\
& \geq \nu_{0}(x)-\nu_{0}(y) .
\end{aligned}
$$

Next consider the case where $\operatorname{coht}_{\widehat{P}} x>r-\nu(-\infty)+\nu(x)$. This happens only when $x \in P$. Therefore $\operatorname{coht}_{\widehat{P}} y=\operatorname{coht}_{\widehat{P}} x-1$ by assumption. Since

$$
r-\nu(-\infty)+\nu(y)<r-\nu(-\infty)+\nu(x) \leq \operatorname{coht}_{\widehat{P}} x-1,
$$

we see that

$$
\operatorname{coht}_{\widehat{P}} y>r-\nu(-\infty)+\nu(y)
$$

Therefore

$$
\nu_{0}(y)=\operatorname{coht}_{\widehat{P}} y=\operatorname{coht}_{\widehat{P}} x-1=\nu_{0}(x)-1<\nu_{0}(x)
$$

and

$$
\nu_{0}(x)-\nu_{0}(y)=1 \leq \nu(x)-\nu(y) .
$$

So we see the claim.

We see that the results of Theorem 3.3 and Lemma 3.4 are also valid if $\{y \in P \mid y \leq x\}$ is pure for any $x \in P$, by using height instead of coheight.

\section{Schubert cycles are level}

Now we fix a field $K$ and integers $m$ and $n$ with $1 \leq m \leq n$.

For an $m \times n$ matrix $M$ with entries in a $K$-algebra $S$, we denote by $K[M]$ the $K$-subalgebra of $S$ generated by all the entries of $M$ and by $G(M)$ the $K$-subalgebra of $S$ generated by all maximal minors of $M$. We also denote by $\Gamma(M)$ the set of all maximal minors of $M$.

It is known that the homogeneous coordinate ring of the Grassmann variety $G_{m}(V)$ of $m$-dimensional subspaces of an $n$-dimensional $K$-vector space $V$ is $G(X)$, where $X$ is an $m \times n$ matrix of indeterminates. It is known that $G(X)$ is an ASL over $K$ generated by $\Gamma(X)$, where we identify $\Gamma(X)$ with a 
combinatorial object $\left\{\left[c_{1}, \ldots, c_{m}\right] \mid 1 \leq c_{1}<\cdots<c_{m} \leq n\right\}$ and define the order of $\Gamma(X)$ by

$$
\left[c_{1}, \ldots, c_{m}\right] \leq\left[d_{1}, \ldots, d_{m}\right] \stackrel{\text { def }}{\Longleftrightarrow} c_{i} \leq d_{i} \text { for } i=1, \ldots, m
$$

Let $0=V_{0} \subsetneq V_{1} \subsetneq \cdots \subsetneq V_{n}=V$ be a complete flag of subspaces of $V$ and let $a_{1}, \ldots, a_{m}$ be integers such that $1 \leq a_{1}<\cdots<a_{m} \leq n$. Then the Schubert subvariety $\Omega\left(a_{1}, \ldots, a_{m}\right)$ of $G_{m}(V)$ is defined by

$$
\Omega\left(a_{1}, \ldots, a_{m}\right):=\left\{W \in G_{m}(V) \mid \operatorname{dim}\left(W \cap V_{a_{i}}\right) \geq i \text { for } i=1, \ldots, m\right\} .
$$

If we put $b_{i}=n+1-a_{m+1-i}$ for $i=1, \ldots, m, \gamma=\left[b_{1}, \ldots, b_{m}\right]$ and $\Gamma(X ; \gamma)=\{\delta \in \Gamma(X) \mid \delta \geq \gamma\}$, then the homogeneous coordinate ring of the Schubert variety $\Omega\left(a_{1}, \ldots, a_{m}\right)$ (Schubert cycle for short) is

$$
G(X ; \gamma):=G(X) /(\Gamma(X) \backslash \Gamma(X ; \gamma)) G(X)
$$

This ring is a graded ASL over $K$ generated by $\Gamma(X ; \gamma)$ ([DEP], $\mathrm{BV}])$. It is also known that $G(X ; \gamma) \simeq G\left(U_{\gamma}\right)$, where $U_{\gamma}$ is the following $m \times n$ matrix with independent indeterminates $U_{i j}[\mathrm{BV}$.

$$
\left(\begin{array}{cccccccccccc}
0 & \cdots & 0 & U_{1 b_{1}} & \cdots & U_{1 b_{2}-1} & U_{1 b_{2}} & \cdots & \cdots & U_{1 b_{m}} & \cdots & U_{1 n} \\
0 & \cdots & 0 & 0 & \cdots & 0 & U_{2 b_{2}} & \cdots & \cdots & U_{2 b_{m}} & \cdots & U_{2 n} \\
\cdots & \cdots & \cdots & \cdots & \cdots & \cdots & \cdots & \cdots & \cdots & \cdots & \cdots & \cdots \\
0 & \cdots & 0 & 0 & \cdots & 0 & 0 & \cdots & 0 & U_{m b_{m}} & \cdots & U_{m n}
\end{array}\right)
$$

We denote the isomorphism $G(X ; \gamma) \stackrel{\sim}{\rightarrow} G\left(U_{\gamma}\right)$ by $\Phi$.

Now we introduce a diagonal term order on the polynomial ring $K\left[U_{\gamma}\right]$. That is, a monomial order on $K\left[U_{\gamma}\right]$ such that the leading monomial of any non-zero minor of $U_{\gamma}$ is the product of the main diagonal of it. For example, the degree lexicographic order induced by $U_{1 b_{1}}>U_{1 b_{1}+1}>\cdots>U_{1 n}>$ $U_{2 b_{2}}>U_{2 b_{2}+1}>\cdots>U_{m n}$.

Then it is easy to see that for standard monomials (of an ASL) $\mu$ and $\mu^{\prime}$ on $\Gamma(X ; \gamma)$ with $\mu \neq \mu^{\prime}, \operatorname{lm}(\Phi(\mu)) \neq \operatorname{lm}\left(\Phi\left(\mu^{\prime}\right)\right)$. So $\Phi(\Gamma(X ; \gamma))$ is a sagbi basis of $G\left(U_{\gamma}\right)$, since $\{\Phi(\mu) \mid \mu$ is a standard monomial on $\Gamma(X ; \gamma)\}$ is a $K$-vector space basis of $G\left(U_{\gamma}\right)$. And $\{\operatorname{lm}(\Phi(\mu)) \mid \mu$ is a standard monomial on $\Gamma(X ; \gamma)\}$ is a $K$-vector space basis of the initial subalgebra in $G\left(U_{\gamma}\right)$ of $K\left[U_{\gamma}\right]$. Note also that $\operatorname{lm}(\Phi(\alpha)) \operatorname{lm}(\Phi(\beta))=\operatorname{lm}(\Phi(\alpha \wedge \beta)) \operatorname{lm}(\Phi(\alpha \vee \beta))$ for any $\alpha, \beta \in \Gamma(X ; \gamma)$. So in $G\left(U_{\gamma}\right)$ is the Hibi ring $\mathcal{R}_{K}(\Gamma(X ; \gamma))$.

Since the poset of all join-irreducible elements of $\Gamma(X ; \gamma)$ is antiisomorphic to a finite poset ideal of $\boldsymbol{N} \times \boldsymbol{N}$ with the componentwise order Miy, we see by Theorem 3.3 that in $G\left(U_{\gamma}\right)$ is a level ring.

Now by a standard deformation argument, we see the following 
Lemma 4.1 Let $A$ be a $K$-subalgebra of a polynomial ring $K\left[Y_{1}, \ldots, Y_{s}\right]$ with finite homogeneous sagbi basis $f_{1}, \ldots, f_{r}$. Let $S=K\left[X_{1}, \ldots, X_{r}\right]$ be the polynomial ring with $r$ variables over $K$. We make $A$ and in $A S$-algebras by $K$-algebra homomorphisms $X_{i} \mapsto f_{i}$ and $X_{i} \mapsto \operatorname{lt}\left(f_{i}\right)$ respectively. Then

$$
\operatorname{dim}_{K} \operatorname{Tor}_{i}^{S}(A, K)_{j} \leq \operatorname{dim}_{K} \operatorname{Tor}_{i}^{S}(\text { in } A, K)_{j}
$$

for any $i$ and $j$.

Since $A$ and in $A$ have the same Hilbert function, $A$ is Cohen-Macaulay, level or Gorenstein if so is in $A$. Therefore we see the following

Theorem 4.2 Schubert cycles are level.

\section{References}

[Bir] Birkhoff, G.: "Lattice Theory." Third ed., Amer. Math. Soc. Colloq. Publ. No. 25, Amer. Math. Soc. Providence, R. I. (1967)

[BV] Bruns, W. and Vetter, U.: "Determinantal Rings." Lecture Notes in Mathematics 1327 Springer (1988)

[DEP] DeConcini, C., Eisenbud, D. and Procesi, C.: "Hodge Algebras." Astérisque 91 (1982)

[Hib] Hibi, T.: Distributive lattices, affine smigroup rings and algebras with straightening laws. in "Commutative Algebra and Combinatorics" (M. Nagata and H. Matsumura, ed.), Advanced Studies in Pure Math. 11 North-Holland, Amsterdam (1987), 93-109.

[Hoc] Hochster, M.: Rings of invariants of tori, Cohen-Macaulay rings generated by monomials and polytopes. Ann. of Math. 96 (1972), 318-337

[Miy] Miyazaki, M.: On the generating poset of Schubert cycles and the characterization of Gorenstein property. to appear in Bulletin of Kyoto University of Education.

[Sta1] Stanley R. P.: Cohen-Macaulay Complexes. in Higher Combinatorics (M.Aigner, ed.), Reidel, Dordrecht and Boston, (1977), 51-62.

[Sta2] Stanley, R. P.: Hilbert Functions of Graded Algebras. Adv. Math. 28 (1978), 57-83. 